\title{
Different degrees of small bowel involvement in peritoneal carcinomatosis may predict the completeness of cytoreduction score. is there a role for staging laparoscopy?
}

\begin{abstract}
The most validated predictors of outcome in peritoneal carcinomatosis (PC) patients undergoing cytoreductive surgery (CRS) and hyperthermic intraoperative chemotherapy (HIPEC) are preoperative tumor burden measured in terms of the peritoneal cancer index (PCI) and completeness of cytoreduction score (CCS), both described by Sugar baker. Intestinal involvement in PC is very common with different levels of invasion during the natural progress of the disease. Regarding patient selection, better assessment of resectability is needed preoperatively. Staging laparoscopy seems to be a feasible and reasonable modality to assess tumor load and tumor biology in the preoperative setting.
\end{abstract}

Volume 8 Issue I - 2017

\author{
Halkia E,' Chrelias G,' Chrelias Ch,' Esquivel \\ $J^{2}$ \\ 'Third Department of Gynecology, University Teaching Hospital \\ "Attikon", National and Kapodistrian University of Athens, \\ Medical School, Athens, Greece \\ ${ }^{2}$ General and Oncology Surgery Clinic, Frederick Memorial \\ Hospital, Frederick, Maryland, USA
}

Correspondence: Halkia E,Third Gynecologic Clinic, University Teaching Hospital Attikon, National and Kapodistrian University of Athens, Medical School, Athens, Greece, Emailevgeniahalkia@gmail.com

Received: August 15, 2017 | Published: November 08, 2017

\section{Introduction}

The successful treatment of peritoneal surface malignancy requires a comprehensive management plan that utilizes CRS and perioperative chemotherapy (POC). In addition, proper patient selection is mandatory. Complete resection of all visible malignancy is essential for the treatment of peritoneal surface malignancy to result in long term survival. Visceral resections and peritonectomy procedures must be used to adequately resect all visible evidence of disease, with their use depending on the distribution and extent of invasion of the malignancy disseminated within the peritoneal space. Normal peritoneum is not excised, only that which is infiltrated by cancer is removed. ${ }^{1}$ Preoperative evaluation should assess whether optimal or complete CRS is feasible in the individual patient. Widespread and high-volume peritoneal disease especially with extensive involvement of small bowel and small bowel mesentery, more than one bowel stenosis /obstructions, are suggestive of aggressive biologic behaviour, lower probability of optimal or complete CRS and poor outcome..$^{2-6}$

\section{Anatomic considerations in small bowel and small bowel mesentery involvement in PC}

The jejunum and ileum measure approximately $6-7 \mathrm{~m}$ in length with the jejunum measure approximately $40 \%$ and the ileum accounting for the distal $60 \%$ of small bowel. There is no sharp demarcation between the jejunum and the ileum. However, there are certain anatomic characteristics that aid the surgeon in distinguishing between the two:

a) The jejunum has a thicker wall because the circular folds or plicae circulars are larger and well developed in the proximal end of the small bowel. These folds are small in the superior part of the ileum and absent in the terminal ileum.

b) The jejunum is of greater diameter. c) The jejunal mesentery contains less fat than that of the ileum and the arterial arcades are easier to visualize than the ileum.

d) The jejuna arterial arcades are simple or double, with long vasa recta compared with the ileum which has four or five arcades and shorter vasa recta.

The superior mesenteric artery (SMA) provides the entire blood supply to the jejunum and ileum. The common pancreatoduodenal artery is the first branch given off and supplies the retroperitoneal duodenum. The remaining small intestine is supplied by the jejunal-ileal arteries. These consist of 10-16 branches that arise from the concave side of the SMA. These branches extend into the mesentery, where they form arcades. From these arcades the vasa recta arise and pass to the mesenteric border of the bowel without anastomosing with one another. The avascular spaces are called the windows of Deaver. The vasa recta continue to form the subserosal plexus. These are sufficient to supply $6-8 \mathrm{~cm}$ of small intestine if the adjacent vasa recta have been occluded or ligated. The space of Treves is an avascular space between the SMA and the ileocolic artery that may result in an inconsistent blood supply to the terminal ileum. Venous drainage of the small intestine is composed of direct tributaries that correspond to the branches of SMA to form the superior mesenteric vein. This joins the splenic vein to form the portal vein. Lymphatic drainage of the small intestine begins in the lacteals of the mucosa villi. These join in the bowel wall and drain through the lymphatic channels that follow the veins. The drainage route begin with the mesenteric lymph nodes which drain to the superior mesenteric lymph nodes, the left lumbar lymphatic trunk and ending in the cistern chilli. ${ }^{7,8}$

\section{Types of small bowel involvement in PC}

Intestinal involvement in PC is very common and although there is a tendency to spread along mesenteric peritoneal and bowel serosal surfaces, it can also invade directly into the visceral wall. The 
histological features and the depth of invasion of the different tumors into the bowel wall in PC are not uniform. Based on the extent of the invasion, the size of the tumor nodule and its anatomic location on the bowel wall, small bowel involvement has been classified into five types, according to Sugar baker:

\section{a) TYPE I: Non-invasive nodules}

Minute nodules of aggressive histology that have not invaded past the peritoneum because of their small size. Also large non-invasive nodules of diffuse peritoneal adenomucinosis.

b) TYPE II: Small invasive nodules on the anti-mesenteric portion of the small bowel.

Nodules that do not separate from the muscular layer of the small bowel.

c) TYPE III: Moderately sized invasive nodules on the antimesenteric portion of the small bowel.

Larger nodules that do not require a full-thickness elliptical resection of the anti-mesenteric portion of the bowel wall.

d) TYPE IV: Small invasive nodules at junction of small bowel and its mesentery.

e) TYPE V: Large invasive nodules.

Nodules that are large and require a segmental small bowel resection with enormous proximal and distal margins on the bowel wall and on the mesentery.

There are two main indications for performing an intestinal resection in $\mathrm{PC}$.

\section{To achieve an optimal volume of residual disease.}

\section{To relieve obstruction.}

Many patients presenting with PC bowel obstruction have previously been treated with multiple chemotherapy regimens or radiotherapy and have limited therapeutic options. Survival of patients with malignant bowel obstruction in the absence of any intervention is usually less than 3 months. ${ }^{9,10}$ Carcinomatosis ileus is a non-obstructive condition characterized by the lack of efficient peristalsis due to carcinomatosis coating the bowel serosa and mesentery. Mechanical bowel obstruction in patients with progressive PC is usually due to malignancy but a "benign" etiology, such as adhesions or radiation stricture, is the cause in $9 \%$ to $23 \%$ of cases. Risk factors for bowel obstruction include multiple prior surgeries and prior radiotherapy. ${ }^{11,12}$ The decision for radicality of the surgical intervention must take into account the likely findings at surgery and probability of successful intervention. It is clear that the overall prognosis for most patients presenting with major malignant bowel involvement is grim.

\section{Diagnostic methods commonly used in the pre- operative estimation of small bowel involvement in PC}

Small bowel contrast studies are generally more accurate $(70 \%-$ $100 \%$ ) than plain film studies in diagnosing small bowel invasion or obstruction. An upper gastrointestinal series with small bowel follow through requires administration of barium or water soluble contrast either orally or via nasogastric tube. Consecutive plain films at progressive time intervals are obtained to identify the site of bowel invasion/ obstruction. Failure of contrast to reach the colon within 24 hours is generally consistent with high-grade or complete obstruction. An enteroclysis study is a modification of the small bowel follow through in which the duodenum is first intubated under fluoroscopy and contrast injected under pressure. This study is highly reliable for both cases, of low and high-grade obstruction because it bypasses the stomach where washout of contrast can occur. ${ }^{13-16}$ Although modern CT technology has significantly contributed to the determination of the PC score, it is a relatively static technology, unable to accurately observe the bowel movement and dynamic distribution of the small intestine. On the other hand, techniques for the observation of the bowel movement under X-ray screen such as barium sulfate swallowing and air-barium sulfate double-contrast studies are not suitable for PC patients as these patients usually have intestinal obstruction and barium sulfate swallow may exacerbate the clinical symptoms of PC. Therefore, a suitable alternative is required.$^{17} \mathrm{Good}$ quality cross-sectional imaging assessment of all components of the PCI score yields the best correlation with actual surgical findings. Although this way the pre-operative assessment of a PCI $>20$ is reasonably accurate, using this cut off to assess respectability is problematic as almost half of these patients are still able to undergo adequate cytoreduction. Better assessment of resectability is needed preoperatively either by refinement of the PCI criteria or by routine staging laparoscopy.

\section{Diagnosing/staging laparoscopy in the pre-operative assessment of patients undergoing CRS + HIPEC for peritoneal surface malignancies}

Despite development of algorithms to predict the ability to achieve optimal cytoreduction (which include tumor markers, disease location, volume of ascites, tumor burden, presence or absence of pleural effusions), it is often difficult to prospectively determine which patients will be left with small volume residual disease and which will have bulky disease after CRS. For this reason, laparoscopy has been suggested to evaluate resectability to the point of no visible tumor prior to laparotomy. Fagotti et al. ${ }^{19}$ used the specific sites of disease determined by laparoscopy to calculate the predictive index of successful cytoreduction or to determine which patients would undergo unnecessary exploration..$^{19}$ Vergote et al. ${ }^{20}$ described 77 patients with clinical and radiological findings predictive of unresectable advanced ovarian cancer PC, by imaging criteria that underwent laparoscopy to evaluate the ability for cytoreduction. ${ }^{20}$ Laparoscopy was demonstrated to be safe and primary optimal cytoreduction was accomplished in $79 \%$ of the patients who were subjected to laparotomy after their laparoscopic assessment. The Society of Gynecologic Oncologists presented 228 cases of advanced ovarian cancer PC that were evaluated with laparoscopy for diagnosis and respectability. ${ }^{21}$ Of the patients with advanced ovarian cancer PC, 90\% were deemed operable and underwent optimal cytoreduction. It is widely known that the most validated predictors of outcome in PC patients undergoing CRS + HIPEC are pre-operative tumor burden, measured in terms of the PCI and CCS. ${ }^{22-24}$ Patients undergoing CRS + HIPEC in which surgery achieved complete or near complete cytoreduction (CC-0 or CC-1) were shown to have significantly greater survival benefit over those who did not. ${ }^{25}$ Survival analysis studies have shown that patients with PCI scores less than 19 (colorectal) and 10 (gastric) benefit most of CRS + HIPEC, facilitating the use of these scores as general cutoffs for excluding patients from this morbid procedure. ${ }^{26-29}$ Exclusion of patients from definitive resection at laparotomy is undesirable and may impede the timely enrollment of patients into alternate therapies. ${ }^{30}$ Diagnostic laparoscopy should be done concomitantly at the time of planned resection or as a separate staging procedure. 
In addition this may permit earlier enrollment of these patients into palliative or neoadjuvant therapy with potential impact on survival. ${ }^{31}$ Since PC presents an advanced stage cancer, visualization may be difficult due to adhesions from the cancer or prior surgical procedures. Additionally there is a potentially increased risk of complications, such as visceral perforations in the setting of PC. All these parameters need to be thoroughly explained to the patient and certainly to be taken under serious consideration by the surgical team..$^{32,33}$

\section{Technique for diagnostic laparoscopy}

Usually the site for the first port placement during diagnostic laparoscopy is decided at the surgeon's discretion based on imaging and clinical findings of the patient. The preferred technique for first creation of pneumoperitoneumis via the optical access technique in the left upper quadrant. In other cases a Hasson's technique is used to establish pneumoperitoneum. After ruling out significant adhesions at the anterior abdominal wall, systemic visual examination of the abdomen is performed to generate the PCI score. Systemic abdominal examination includes also special attention to per-splenic, perihepatic, sub-diaphragmatic, pelvic, omental bursa and bowel tumor load. . $^{34-36}$

\section{Conclusion}

Advanced stage PC, commonly involves the serosal surfaces of the small bowel and it's mesentery at different levels of invasion during the natural progression of the abdominal carcinomatosis and the aggressiveness of the tumor cell. Localized / limited superficial disease may be amenable to sharp excision or electrocautery ablation, however, segmental resection of the small bowel is often necessary as part of maximal cytoreductive surgical effort. Abdominal carcinomatosis may spread to the bowel mesentery as miliary disease or tumor plaques covering the peritoneal surface or as regional lymphadenopathy. The decision to attempt cytoreduction should be based on the overall extent of tumor and the likely hood of achieving an optimal volume of residual disease. Staging laparoscopy serves as a reasonable mechanism to assess resectability in a patient with PC prior to laparotomy. In patients where optimal cytoreduction is thought not to be feasible, a biopsy can be performed to confirm the clinical impression of PC and other therapeutic modalities ie. chemotherapy can be initiated quickly.

\section{Conflicts of interest}

None.

\section{Acknowledgements}

None.

\section{References}

1. Sugarbaker PH. Peritonectomy procedures. Surg Oncol Clin N Am 2003;12(3):703727

2. Piso P, Glockzin G, von Breitenbuch P, et al. Patient selection for a curative approach to carcinomatosis. Cancer. 2009;J 15(3):236-242.

3. Salani R, Zahurak ML, Santillan A, et al. Survival impact of multiple bowel resections in patients undergoing primary cytoreductivesurgery for advanced ovarian cancer: a case-control study. Gynecol Oncol. 2007;107(3):495-499.

4. Cai HB, Zhou YF, Chen HZ, et al. The role of bowel surgery with cytoreduction for epithelial ovarian cancer. Clin Oncol ( $R$ Coll Radiol). 2007;19(10):757-762.
5. Gillette-Cloven N, Burger RA, Monk BJ, et al. Bowel resection at the time of primary cytoreduction for epithelial ovarian cancer. Am Coll Surg. 2001;196(6):626-632.

6. Jaeger W, Ackermann S, Kessler H, et al. The effect of bowel resection on survival in advanced epithelial ovarian cancer. Gynecol Oncol. 83(2):286-291.

7. Bristow RE, Karlan BY, Chi DS. Surgery for ovarian cancer. Principles and practice. 2nd edn. Informa Healthcare pg. 141.

8. Hoffman MS, Griffin D, Tebes S, et al. Sites of bowel resected to achieve optimal ovarian cancer cytoreduction: implications regarding surgical management. Am J Obstet Gynecol. 2005;193(2):582-586.

9. Tunca JC, Buchler DA, Mack EA, et al. The management of ovarian cancer caused bowel obstruction. Gynecol Oncol. 1981;12(Pt 1):186192.

10. Rubin SC, Hoskins WJ, Benjamin I, et al. Palliative surgery for intestinal obstruction in advanced ovarian cancer. Gynecol Oncol. 1989;34(1):1619.

11. Clarke-Pearson DL, Chin NO, DeLong ER, et al. Surgical management of intestinal obstruction in ovarian cancer. Clinical features, postoperative complications and survival. Gynecol Oncol. 1987;26(1):11-18.

12. Redman CW, Shafi MI, Ambrose S, et al. Survival following intestinal obstruction in ovarian cancer. Eur J Surg Oncol. 1988;14(5):383-386.

13. Ericksen AS, Krasna MJ, Mast BA, et al. Use of gastrointestinal contras studies in obstruction of the small bowel and large bowel. Dis Colon Rectum. 1990;33(1):56-64.

14. Anderson CA, Humphrey WT. Contrast radiography in small bowel obstruction: a prospective randomized trial. Mil Med. 1997;162(11):749 752.

15. Shrake PD, Rex DK, Lappas JC, et al. Radiographic evaluation of suspected small bowel obstruction. Am J Gastroenterol. 1991;86(2):175178

16. DiSantis DJ, Ralls PW, Balfe DM, et al. The patient with suspected small bowel obstruction: imaging strategies. American College of Radiology. ACR Appropriateness Criteria. Radiology. 2000;215(suppl):121-124.

17. Mei LJ, Wang LW, Huang CQ, et al. Oral gastrograpfy for evaluation of the functional impact of peritoneal carcinomatosis: Correlation with clinicopathological findings. Mol and Clin Oncol. 2015;3(5):979986.

18. Flicek K, Ashfaq A, Johnson CD, et al. Correlation of Radiologic with Surgical Peritoneal Cancer Index Scores in Patients with Pseudomyxoma Peritonei and Pertoneal Carcinomatosis: How well Can We Predict Resectability? J Gastrointest Surg. 2016;20(2):307-312.

19. Fagotti A, Ferrandina G, Fanfani F, et al. Prospective validation of a laparoscopic predictive model for optimal cytoreductionin advanced ovarian carcinoma. Am J Obstet Gynecol. 2008;199(6):642.

20. Vergote I, De Wever I, Tjalma W, et al. Neoadjuvant chemotherapy or primary debulking surgery in advanced ovarian carcinoma: a retrospective analysis of 285 patients. Gynecol Oncol. 1998;71(3):431436.

21. Vergote I. Ovarian cancer neoadjuvantchemotherapy. San Antonio, Texas, USA. Society of Gynecologic Oncologists; 2009.

22. Harmon RL, Sugarbaker PH. Prognostic indicators in peritoneal carcinomatosis from gastrointestinal cancer. Int Semin Surg Oncol. 2005;2(1):3.

23. Yonemura Y, Canbay E, Ishibashi H. Prognostic factors of peritoneal metastases from colorectal cancer following cytoreductive surgery and perioperative chemotherapy. Scientific World J. 2013:978394. 
24. Van Sweringen HL, Hanseman DJ, Ahmad SA, et al. Predictor of survival in patients with high-grade peritoneal metastases undergoing cytoreductive surgery and hyperthermic intraperitoneal chemotherapy. Surgery. 2012;152(4):617-624.

25. Yang XJ, Huang CQ, Suo T, et al. Cytoreductive surgery and hyperthermic intraperitoneal chemotherapy improves survival of patients with peritoneal carcinomatosis from gastric cancer: final results of a phase III randomized clinical trial. Ann Surg Oncol. 2011;18(6):1575-1581.

26. Bakrin N, Gilly FN, Baratti D, et al. Primary peritoneal serous carcinoma treated by cytoreductive surgery combined with hyperthermic intraperitoneal chemotherapy. A multi-institutional study of 36 patients. Eur J Surg Oncol. 2013;39(7):742-747.

27. Quenet F, Goere D, Mehta SS, et al. Results of two bi-institutiona prospective studies using intraperitoneal oxaliplatin with or without irinotecan during HIPEC after cytoreductive surgery for colorectal carcinomatosis. Ann Surg. 2011;254(2):294-301.

28. Wu XJ, Yuan P, Li ZY, et al. Cytoreductive surgery and hyperthermic intraperitoneal chemotherapy improves the survival of gastric cancer patients with ovarian metastasis and peritoneal dissemination. TumourBiol. 2013;34(1):463-469.

29. Yonemura Y, Elnemr A, Endou Y, et al. Effects of neoadjuvant intraperitoneal/systemic chemotherapy (bidirectional chemotherapy) for the treatment of patients with peritoneal metastasis from gastric cancer. Int J SurgOncol. 2012:148420.
30. Society of American Gastrointestinal and Endoscopic Surgeons: Guidelines for Diagnostic Laparoscopy. 2010.

31. Chang L, Stefanidis D, Richardson WS, et al. The role of staging laparoscopy for intraabdominal cancers: an evidence-based review. Surg Endosc. 2009;23(2):231-241.

32. Seshadri RA, Hemanth RE. Diagnostic laparoscopy in the pre-operative assessment of patients undergoing cytoreductive surgery and HIPEC for peritoneal surface malignancies. Indian J Surg Oncol. 2016;7(2):230 235

33. Sommariva A, Zagonel V, Rossi CR. The role of laparoscopy in peritoneal surface malignancies selected for hyperthermic intraperitoneal chemotherapy (HIPEC) Ann Surg Oncol. 2012;19(12):3737-3744.

34. Jayakrishnan TT, Zacharias AJ, Sharma A, et al. Role of laparoscopy in patients with peritoneal metastases considered for cytoreductive surgery and hyperthermic intraperitoneal chemotherapy (HIPEC). World J Surg Oncol. 2014;12:270

35. Iversen LH, Rasmussen PC, Lauberg S. Value of laparoscopy before cytoreductive surgery and hypothermic intraperitoneal chemotherapy for peritoneal carcinomatosis. BrJSurg. 2013;100(2):285292.

36. Najah H, Lo Dico $R$, Grienay $M$, et al. Single-incision flexible endoscopy (SIFE) for detection and staging of peritoneal carcinomatosis. Surg Endosc. 2015;30(9):3808-3815. 